\title{
Decomposition-coordination model and algorithm for parallel calculation of power system state estimation problem
}

\author{
Mashauri Adam Kusekwa \\ Electrical Engineering department, Dar es Salaam Institute of Technology (DIT), Dar es Salaam-Tanzania
}

Email address:

kusekwa_adam@yahoo.com,Kusekwa_adam@dit.ac.dit

To cite this article:

Mashauri Adam Kusekwa. Decomposition-Coordination Model and Algorithm for Parallel Calculation of Power System State Estimation Problem. American Journal of Electrical Power and Energy Systems. Vol. 3, No. 6, 2014, pp. 107-118. doi: 10.11648/j.epes.20140306.12

\begin{abstract}
Power system state estimation is the process of computing a reliable estimate of the system state vector composed of bus voltages' magnitudes and angles from telemetered measurements on the system. This estimate of the state vector provides the description of the system necessary for operation, security monitoring and control. Many methods are described in literature for solving the state estimation problem, the most important of which are the classical weighted least squares and the non-quadratic method. However, both showed drawbacks when it comes to application to large-scale power system networks. In this paper, a new method in the name of decomposition-coordination approach using the weighted least squares is introduced in solving the large-scale power system state estimation problem. The estimation criterion is reformulated; voltage measurement, real and reactive power injections, real and reactive power flows, and real and reactive power flows in tie-line models of a decomposed system are developed. Two level structure of solving the estimation problem is introduced. The first level solves the sub-problem using gradient procedure methods while the second level determines the interconnection variables using predictive method. The positive characteristic of the method is that the coordinator has little work of predicting interconnection variables instead of solving the state estimation problem. The method can be used to solve a multi-area state estimation using parallel or distributed processing architectures.
\end{abstract}

Keywords: Power Systems, Modelling of Measurement Data, State Estimation, Decomposition-Coordination Method, Algorithm

\section{Introduction}

The heart of the data processing activities at electrical utility central dispatch centre (CDC) is the power system state estimator using both real-time measurements and historical database. The state estimator detects and identifies errors in the measurements and computes an optimal estimate of the system state vector of bus voltages' magnitudes and angles. This optimal estimate is then used by the security monitoring, operation and control functions [1-5]. The state estimation process is based on a statistical criterion that estimates the true value of the state vector of the system to minimize the selected criterion [6-8].

The most common and familiar criterion used in power industry is the weighted least square method where the objective function is to minimize the sum of the squares of the difference between each measured value and the true estimated value with each squared difference divided or weighted by the variance of meter error[9-10].

State estimation can eliminate the effect of bad data [12] and allow the temporary loss of measurements without significant affecting the quality of the estimated values. It is used to filter redundant data, eliminate incorrect measurements, it allows determination of the power flows in part of the network that are not directly metered and it can produce reliable state estimate

Nowadays, system networks are becoming more and more complicated. In this aspect monitoring and control of these networks feel the necessity of having robust and scalable methods for state estimation that maintain performance of large-scale systems. Recently, there has been increasing interest in improving various types of state estimation algorithms used in the industry [11] to make them applicable in the ever expanding systems. These improved state 
estimation algorithms have been implemented in various power system central dispatch centres using a centralized estimation algorithm. In this set ups measurements from all sensors are sent to a central estimation unit where the state of the whole system is estimated. Centralized processing has posed challenges such as problems relating to communication between the sensors and the central estimation unit and processing facility (computer) memory limits. These challenge motivated researchers to move toward finding effective, robust and reliable techniques of processing state estimation of large-scale power system networks.

There are two approaches to carry out large-scale power system state estimation. First is to decompose the system into sub-systems and model the neighboring utilities in detail and accurately in one's own state estimator. Second, is to obtain the state estimation output from each sub-system and convert them into global estimation; this set up is known as hierarchical state estimation.

Hierarchical method has been investigated in the past with the aim of reducing computation time, memory requirements and amount of data exchange between sub-systems. VanCutsem et al. [12-14] proposed a two-level state estimation algorithm by dividing the system into known overlapping sub-systems which are connected by tie-lines. In the first level each sub-system performs state estimation independently with respect to data and information available in the sub-system. In the second level, the boundary bus states are re-estimated and all voltage angles are coordinated to a global reference.

In [15] a two-level state estimator for multi-area interconnected system is proposed. In the first level of the algorithm, each area runs their own state estimator using measurements from its own area. In the second level of the algorithm, the central coordinator collects the state estimation from each area and coordinates them to get the multi-area state estimation with respect to global reference. In this way the coordinator can use the measurements available from the boundary network such as tie-line power flows, boundary bus injections, boundary bus voltage etc. The coordinator can also use the boundary bus state available from each area state estimators as pseudo measurements to increase the redundancy.

Aguado et al. [16] addressed power system state estimation problem using decomposition-coordination techniques. The whole system network is divided into geographical areas. Then, for each area, an area power system state estimation problem is formulated. The global optimum of the overall system is obtained by iteratively coordinating the solution of area state estimation subproblems. By using decomposition-coordination techniques, the integrated optimum solution can be achieved by only sharing a reduced amount of information of tie-lines. The techniques can be applied within a utility with a transmission network spanning over different regions, in such a set up every region dispatch centre perform a state estimation algorithm in coordination with neighboring dispatch centre. In case of a large-scale power system networks where computation is a concern, a distributed implementation can be an alternative to save computation time by simultaneously running many power system state estimation algorithm.

Interesting is the work by Aguado et al. [16]. However, the work presented in this paper is different in implementation of decomposition-coordination method. In this paper, decomposition process is implemented using bus admittance matrix of a power system network instead of geographical areas. In this way the sub-matrices obtained after decomposition represent sub-systems; in case of power system state estimation they represent sub-problems. Then measurement model for voltage magnitude, real and reactive power injections, real and reactive power flows and real and reactive power flows in tie-lines are developed. The state estimation problem is solved using a two-level structure under decomposition-coordination principle proposed in [17]. Advantages of the method is that there is no need of reestimating the boundary bus states; these are included in the real and reactive power injection model, also the method reduces the coordinators work to just calculating of subsystem interconnection variables by using prediction method and sent these variables to the first level to be used as measurements in computing sub-system state estimation.

The paper is organized as follows. Section 2 describes problem formulation of the state estimation problem. Section 3 presents state estimation solution problem formulation and solution under two-level structures. Section 4 presents first and second level algorithms. Section 5 Discusses positive characteristic of the proposed method and its advantages in solving power system state estimation problem. Section 6 concludes the paper.

\section{Problem Formulation}

The power system state estimator processes real-time redundant telemetered from substations and pseudo measurements to provide a complete, coherent and reliable system database, which can describe the current electrical state of the system network [18-19]. The measurements, which include voltage magnitudes, real and reactive power injections and real and reactive line power flows are measured from the network at a certain moment, thus getting an estimate for respective state vector i.e. vector of voltages' magnitude and angles on different buses [20].

Consider an interconnected system decomposed into $\mathrm{N}_{S}$ sub-system shown in Figure 1. Individual sub-system is connected to each sub-system through the tie-line network (Figure 2). The buses in each sub-system can be categorized as internal buses, internal boundary buses and external boundary buses. 


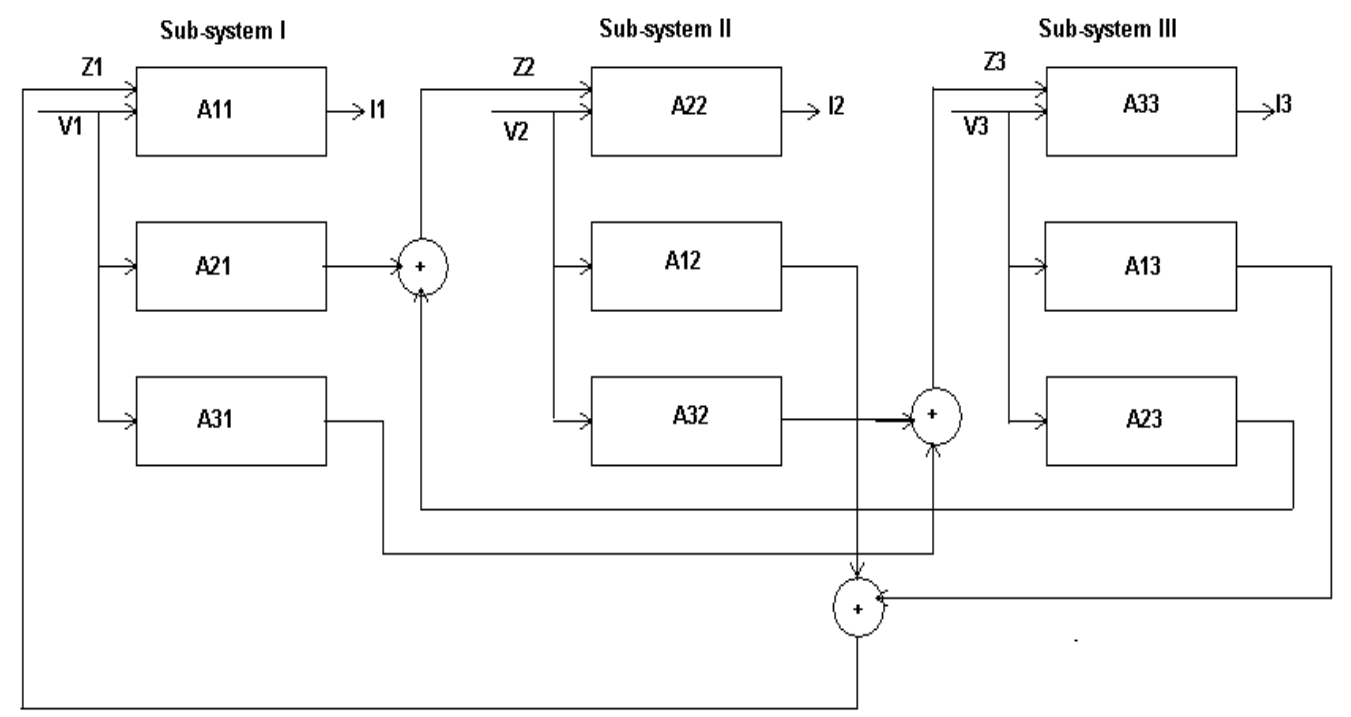

Figure 1. System Decomposed model structure

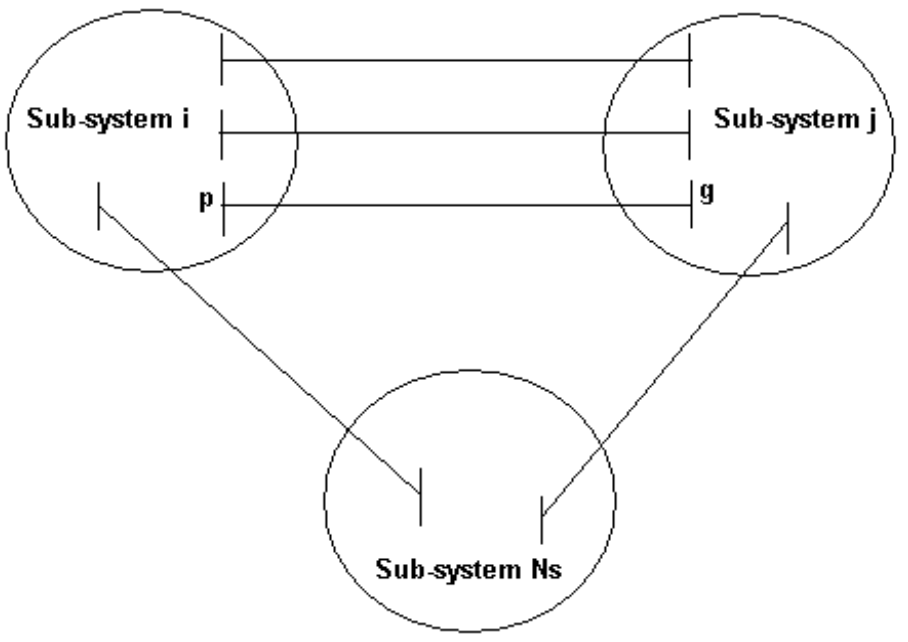

Figure 2. Tie-lines connecting sub-systems

The weighted least squares (WLS) is used in this paper, in this way it is considered that the criterion is a sum of subcriterion determined for every one of the sub-systems. In the common case, the criterion is written as follows:

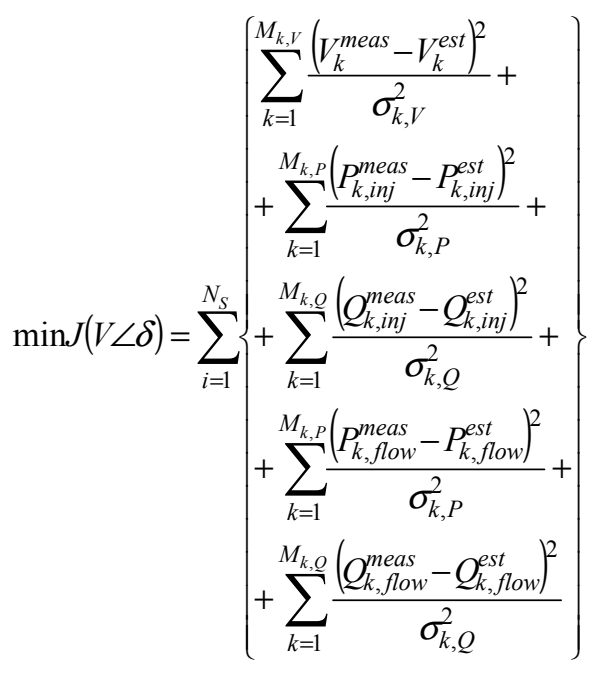

Equation (1) can be written in short form as:

$$
\min J(V \angle \delta)=\sum_{i=1}^{N_{S}} J_{i}(V \angle \delta), i=\overline{1, N_{S}}
$$

Where

$\mathrm{N}_{\mathrm{S}}$ is the number of sub-systems.

$M_{k, V}, M_{k, P}^{i n j}, M_{k, Q}^{i n j}, M_{k, P}^{\text {flow }}, M_{k, Q}^{\text {flow }}$, are the dimension of the corresponding measured variables. The type and number of measurements for different sub-systems can be different. The simplified criterion used in this paper is given by:

$$
\min J(V \angle \delta)=\sum_{i=1}^{N_{S}} \sum_{k=1}^{M_{k, V}} \frac{\left(V_{k}^{\text {meas }}-V_{k}^{e s t}\right)^{2}}{\sigma_{k, V}^{2}}
$$

Where $\sigma$ is the standard deviation, $V_{k}^{\text {meas }}$ : is the measured quantity, $V_{k}^{e s t}$ : is the estimated quantity (calculated) 


\subsection{Measurement Model}

The global data measurement model is given by

$$
z=h(x)+\varepsilon
$$

Where $\varepsilon$ : is the measurement error.

The model has 4 parts determined by the type of measurements. When the power system is considered to be used for decomposed solution of state estimation problem, these 4 parts of the measurement model can be determined in different ways.

\subsubsection{Voltage Magnitude Data Model}

Voltage magnitude measurement model is for the whole system and is given by:

$$
z_{V}=|V|
$$

Where

$$
z_{V} \in \mathfrak{R}^{N}
$$

$\mathrm{N}$ : is the number of model buses.

Decomposition of this model is direct and is determined by the selected number of buses in every sub-system. The ith sub-system model can be written as:

$$
z_{i, V}=\left|V_{i}\right| i=\overline{1, N_{S}}, z_{i, V} \in \Re^{n_{i}}
$$

$n_{i}:$ is the number of buses in the $i t h$ sub-system

\subsubsection{Real and Reactive Power Injection Data Model}

The real and reactive power injection data model for the whole power system is decomposed in [21]. The obtained sub-system model is characterized with local for the subsystem state variables and with disturbance input from other sub-system and is given by:

$$
\begin{gathered}
P_{i}=G_{i}^{R} V_{i}+\sum_{\substack{j=1 \\
j \neq i}}^{N_{S}} G_{i j}^{R} V_{j}=G_{i}^{R} V_{i}+y_{i}^{R} \\
y_{i}^{R}=\sum_{\substack{j=1 \\
j \neq i}}^{N_{S}} G_{i j}^{R} V_{j} i=\overline{1, N_{S}}, j=\overline{1, N_{S}}, j \neq i \\
G_{i}^{R} \in \Re^{n_{i} x n_{i}}, G_{i j}^{R} \in \mathfrak{R}^{n_{i} x n_{j}}, y_{i}^{R} \in \mathfrak{R}^{n_{i}} \\
Q_{i}=G_{i}^{i m} V_{i}+\sum_{\substack{j=1 \\
j \neq i}}^{N_{S}} B_{i j}^{i m} V_{j}=G_{i}^{i m} V_{i}+y_{i}^{i m} \\
y_{i}^{i m}=\sum_{\substack{j=1 \\
j \neq i}}^{N_{S}} B_{i j}^{i m} V_{j}
\end{gathered}
$$

$$
G_{i}^{i m} \in \Re^{n_{i} x n_{i}}, B_{i j}^{i m} \in \mathfrak{R}^{n_{i} x n_{j}}, y_{i}^{i m} \in \mathfrak{R}^{n_{i}}
$$

The type and number of measurements in every subsystem can be different and independent. In the common case the injection data model can be written in matrix form as:

$$
\begin{array}{r}
{\left[\begin{array}{c}
P_{i} \\
Q_{i}
\end{array}\right]=\left[\begin{array}{c}
G_{i}^{R} \\
G_{i}^{i m}
\end{array}\right] V_{i}+\left[\begin{array}{c}
y_{i}^{R} \\
y_{i}^{i m}
\end{array}\right]=G_{i, i n j} V_{i}+y_{i, i n j}} \\
G_{i, i n j} \in \mathfrak{R}^{2 n_{i} x n_{i}}, y_{i, i n j} \in \mathfrak{R}^{2 n_{i}} \\
y_{i, i n j}=\sum_{\substack{j=1 \\
j \neq i}}^{N_{S}}\left[\begin{array}{c}
G_{i j}^{R} \\
B_{i j}^{i m}
\end{array}\right] V_{j}=\sum_{\substack{j=1 \\
j \neq i}}^{N_{S}} h_{i, i n j}\left(V_{i} \angle \delta_{i}, V_{j} \angle \delta_{j}\right)
\end{array}
$$

Eqns (10) and (11) can be written in the notation of data model as:

$$
z_{i, i n j}=h_{i, i n j}\left(V_{i} \angle \delta_{i}\right)+y_{i, i n j}+\varepsilon_{i, i n j}
$$

Where

$$
\begin{array}{r}
h_{i, i n j}\left(V_{i} \angle \delta_{i}\right)=G_{i, i n j} V_{i} \\
y_{i, i n j}=\sum_{\substack{j=1 \\
j \neq i}}^{N_{S}} h_{i, i n j}\left(V_{i} \angle \delta_{i}, V_{j} \angle \delta_{j}\right)
\end{array}
$$

$$
h_{i, i n j} \in \mathfrak{R}^{2 n_{i} x n_{i}}
$$

The dimension of the vectors in Eqn (10) and the matrices have the maximal possible value but the number of measurements can be different. For every of the subproblems a local voltage angle reference bus has to be introduced. In this case the sub-systems are independent.

\subsubsection{Real and Reactive Power Flow Data Model}

Real and reactive power flows is determined for every two interacting buses separately. This means that this model can be directly decomposed according to the selected dimension of the sub-systems. It is supposed for the power flows between thepth and qthbuses in the ith sub-system (see Figure 2) that real and reactive power flows from bus $p$ to bus qare:

$$
\begin{aligned}
P_{i, p q}= & V_{i, p}^{2}\left(g_{i, p q}^{s h}+b_{i, p q}\right)- \\
- & V_{i, p} V_{j, q}\left[g_{i, p q} \cos \delta_{i, p q}-b_{i, p q} \sin \delta_{i, p q}\right] \\
Q_{i, p q}= & -V_{i, p q}^{2}\left(b_{i, p q}^{s h}+b_{i, p q}\right)+ \\
& +V_{i, p} V_{j, q}\left[g_{i, p q} \sin \delta_{i, p q}+b_{i, p q} \cos \delta_{i, p q}\right]
\end{aligned}
$$

The models of the flows from bus $\mathrm{q}$ to bus $\mathrm{p}$ are given as follows: 


$$
\begin{aligned}
P_{i, q p} & =V_{i, q}^{2}\left(g_{i, q p}^{s h}+g_{i, q p}\right)- \\
& -V_{i, q} V_{i, p}\left[g_{i, q p} \cos \delta_{i, q p}-b_{i, q p} \sin \delta_{i, q p}\right] \\
Q_{i, q p}= & -V_{i, q}^{2}\left(b_{i, q p}^{s h}+b_{i, q p}\right)+ \\
& +V_{i, q} V_{i, p}\left[g_{i, q p} \sin \delta_{i, q p}+b_{i, q p} \cos \delta_{i, q p}\right]
\end{aligned}
$$

Where

$$
\begin{aligned}
\delta_{i, p q} & =\delta_{i, p}-\delta_{i, q} \\
\delta_{i, q p} & =\delta_{i, q}-\delta_{i, p}
\end{aligned}
$$

In general the power flow data model can be written as:

$$
\begin{aligned}
& z_{i, f l o w}=\left[\begin{array}{llll}
P_{i, p q}, & Q_{i, p q}, & P_{i, q p}, & Q_{i, q p}
\end{array}\right]^{T}= \\
& =h_{i, \text { flow }}\left(V_{i} \angle \delta_{i}\right)+\varepsilon_{i, \text { flow }} \quad i=\overline{1, N_{S}}
\end{aligned}
$$

Where

$$
z_{i, \text { flow }} \in \mathfrak{R}^{\left(M_{i, P}^{\text {flow }}+M_{i, Q}^{\text {flow }}+M_{i, P}^{\text {flow }}+M_{i, Q}^{\text {flow }}\right)}
$$

The type of the measurements and the number of measurements of real and reactive power flows can be different for different sub-systems.

\subsubsection{Tie-Line Data Model}

The $N_{S}$ sub-systemsshown in Figure 1are connected by tielines (electrical transmission lines or transformers). In this paper only electrical transmission lines are considered. The two ends of each tie-line are buses belonging to different subsystems. The set of these boundary buses define an $\left(N_{S}+1\right)$ th sub-system called interconnection sub-system. The measurement model for the tie-lines between the ith and jth sub-systems, when the number of tie-lines is $N_{t l}$ is given in [21] as follows:

$$
\begin{gathered}
P_{i j, p g}=G_{i j, p g} V_{i, p}^{2}-V_{i, p} V_{j, g} G_{i j, p g} \\
p=\overline{1, N_{t l i j i}}, g=\overline{1, N_{t l, i j}}, i=\overline{1, N_{S}} \\
j=\overline{1, N_{S}}, p \neq g, j \neq i \\
Q_{i j, p g}=-V_{i, p}^{2}\left(B_{i j, p g}+b_{i j, p g}^{s h}\right)+V_{i, p} V_{j, g} B_{i j, p g}
\end{gathered}
$$

The vector of measurements for the ith sub-system can be written as

$$
\begin{gathered}
z_{t l, i j, p g}=\left[\begin{array}{c}
P_{i j, p g} \\
Q_{i j, p g}
\end{array}\right]=\left[\begin{array}{c}
G_{i j, p g} \\
-B_{i j, p g}-b_{i j, p g}^{s h}
\end{array}\right] V_{i, p}^{2}+ \\
+V_{i, p}\left[\begin{array}{c}
G_{i j, p g} \\
-B_{i j, p g}
\end{array}\right] V_{j, g}
\end{gathered}
$$

The vector of measurements for the interconnected system is given by:

$$
z_{t l}=\left\lfloor\begin{array}{llllll}
z_{t l, 1,}^{T}, & z_{t l, 2}^{T}, & \cdots & z_{t l, i}^{T} & \cdots & z_{t l, N_{S}}^{T}
\end{array}\right\rfloor
$$

The measurement model is a non-linear and can be written as:

$$
\begin{aligned}
z_{t l}=h_{t l}\left(V_{i} \angle \delta_{i}, V_{j} \angle \delta_{j}\right) \\
i=\overline{1, N_{S}} \cdot j=\overline{1, N_{S}}, j \neq i
\end{aligned}
$$

It can be observed that the ijth sub-system tie-line flow measurement model has two parts. The first part depends on the ith sub-system voltages while the second part depends on the jth sub-system. This means that the measurement model can be represented as a sum from a model of the ith subsystem and the model of interconnection with other subsystems. The first part depends on the voltage $V_{i, p}$ of the ith sub-system and the second depends on $V_{j, g}$ of the jth subsystem. These voltages Participate also in the injection model of the sub-system of the interconnected system. Included in the vector of voltages of the sub-system are also the border buses and voltages. In this way it is not necessary to calculate again the state estimates of the border injections as they are calculated using the injection data model.

Hence, the model of the tie-line data for the ith sub-system can be written in the following way:

$$
\begin{gathered}
z_{i, t l}=h_{i, t l}\left(V_{i} \angle \delta_{i}\right)+y_{i, t l} \\
y_{i, t l}=h_{i j, t l}\left(V_{j} \angle \delta_{j}\right)
\end{gathered}
$$

The dimension of the measurement vector depends on the number of the tie-lines between the ith sub-system and other sub-system.

Finally the data model(measurement) of the ith sub-system with measurements of the voltage, real and reactive power injections, real and reactive power flows, and real and reactive power flows in the tie-lines can be written as:

$$
\begin{aligned}
& z_{i, V}=V_{i}+\varepsilon_{i, V} \\
& z_{i, i n j}=h_{i, i n j}\left(V_{i} \angle \delta_{i}\right)+y_{i, i n j}+\varepsilon_{i, i n j} \\
& z_{i, \text { flow }}=h_{i, \text { flow }}\left(V_{i} \angle \delta_{i}\right)+\varepsilon_{i, \text { flow }} \\
& z_{i, t l}=h_{i, t l}\left(V_{i} \angle \delta_{i}\right)+y_{i, t l}+\varepsilon_{i, t l}
\end{aligned}
$$

The model given by (28) is used for formulation and solution of state estimation problem under decomposed environment.

\section{State Estimation Problem Solution}

\subsection{Two Level State Estimation Solution}

The solution of a power system state estimation can be obtained by solving the following Lagrangian function 


$$
L=\sum_{i=1}^{N_{S}}\left\{\begin{array}{l}
\frac{\left(V_{i}^{\text {meas }}-V_{i}^{e s t}\right)^{2}}{\sigma_{i, V}^{2}}+ \\
+\rho_{i, i n j}^{T}\left[y_{i, i n j}-\sum_{\substack{j=1 \\
j \neq i}}^{N_{S}} h_{i, i n j}\left(V_{i} \angle \delta_{i}\right)\right]+ \\
+\lambda_{i, i n j}^{T}\left[z_{i, i n j}-h_{i, i n j}\left(V_{i} \angle \delta_{i}\right)-y_{i, i n j}\right]+ \\
+\rho_{i, t l}^{T}\left[y_{i, t l}-h_{i j, t l}\left(V_{i} \angle \delta_{i}, V_{j} \angle \delta_{j}\right)\right]+ \\
+\lambda_{i, t l}^{T}\left[z_{i, t l}-h_{i, t l}\left(V_{i} \angle \delta_{i}\right)-y_{i, t l}\right]
\end{array}\right\}
$$

where

$\rho_{i, i n j}, \rho_{i, t l}, \lambda_{i, i n j}, \lambda_{i, t l}$ are vectors of Lagrange multipliers.

The Lagrangian function includes the criterion and the model of the interconnected equations of the sub-system according to power injections and flows. It can be seen from (29) if the interconnections $h_{i j, i n j}$ and $h_{i j, t l}$ can be decomposed, then the Lagrangian function can be decomposed and the state estimation problem can be solved in a fully decentralized way.

Such a type of decomposition of the Lagrangian function can be achieved if the problem for state estimation is solved in a two-level structure using the principles of decomposition coordination [17]. The mixed principle of prediction of the aims of the sub-system represented by the Lagrange variables $\rho_{i, i n j}$ and $\rho_{i, t l}$, and of prediction of interconnection of the sub-systems $y_{i, i n j}$ and $y_{i, t l}$ is applied to the Lagrangian function of (29). This principle is implemented by introducing a coordinator on the second level of the two-level structure of the solution to the problem. The coordinator predicts the values of the Lagrange variables and interconnections as follows:

$$
\begin{aligned}
& \rho_{i, i n j}=\rho_{i, i n j}^{c} \\
& \rho_{i, t l}=\rho_{i, t l}^{c} \\
& y_{i, i n j}=y_{i, i n j}^{c} \\
& y_{i, t l}=y_{i, t l}^{c}
\end{aligned}
$$

Where $c$ is the index of the coordinating procedure.

Substitution of the coordinating variables given in (30) into the Lagrangian function (29) allows the interconnection terms to be distributed between the sub-systems in the following way [17]

$$
\sum_{i=1}^{N_{S}} \rho_{i, i n j}^{T} \sum_{\substack{j=1 \\ j \neq i}}^{N_{S}} h_{i j, i n j}\left(V_{i} \angle \delta_{i}, V_{j} \angle \delta_{j}\right)=\sum_{i=1}^{N_{S}} \sum_{\substack{j=1 \\ j \neq i}}^{N_{S}} \rho_{j, i n}^{T} h_{j i, i n j}\left(V_{i} \angle \delta_{i}\right)
$$

$$
\sum_{i=1}^{N_{S}} \rho_{i, t l}^{T} h_{i j, t l}\left(V_{i} \angle \delta_{i}, V_{j} \angle \delta_{j}\right)=\sum_{i=1}^{N_{S}} \rho_{j, t l}^{T} h_{j i, t l}\left(V_{i} \angle \delta_{i}\right)
$$

Equations (31) and (32) are possible on the basis that the connection between the primal variables $V_{j}$ and the dual (Lagrange's) variables $\rho_{i}$. As the dual variables have the voltages of other sub-systems can be substituted by the voltages of the ith sub-system and the Lagrange's variables of the $j t h$ sub-system. In this way the Lagrangian function is a function only of the voltages of the ith sub-system and can be fully decomposed. This means that the state estimation can be solved separately. Then, the system solutions in this case depends on the values of the coordinating variables, which means that the optimal solutions will be obtained only when the values of the coordinating variables is computed by an iterative process of coordination based on the necessary conditions for optimality of the Lagrangian function towards the coordinating variables and on the solution of the separate sub-system's problems.

The necessary conditions for optimality of the Lagrangian function towards the coordination variables are given by:

$$
\begin{gathered}
\frac{\partial L}{\partial \rho_{i, i n j}}=y_{i, i n j}^{c}-\sum_{\substack{j=1 \\
j \neq i}}^{N_{S}} h_{i j, i n j}\left(V_{i} \angle \delta_{i}, V_{j} \angle \delta_{j}\right)=0 \\
\frac{\partial L}{\partial y_{i, i n j}}=\rho_{i, i n j}^{c}-\lambda_{i, i n j}=0 \\
\frac{\partial L}{\partial \rho_{i, t l}}=y_{i, t l}^{c}-h_{i, j l}\left(V_{i} \angle \delta_{i}, V_{j} \angle \delta_{j}\right)=0 \\
\frac{\partial L}{\partial y_{i, t l}}=\rho_{i, t l}^{c}-\lambda_{i, t l}=0
\end{gathered}
$$

The value of voltages and the value of Lagrange's variables $\lambda_{i, i n j}$ and $\lambda_{i, t l}$ can be obtained as solutions of the sub-systems state estimation problems.

Equations (33) to (36) can be solved analytically using the solutions obtained from first level sub-problems in the following way:

$$
\begin{gathered}
y_{i, i n j}^{c+1}=\sum_{\substack{j=1 \\
j \neq i}}^{N_{S}} h_{i j, i n j}\left(V_{i}^{c} \angle \delta_{i}^{c}, V_{j}^{c} \angle \delta_{j}^{c}\right) \\
\rho_{i, i n j}^{c+1}=\lambda_{i, i n j}^{c} \\
y_{i, t l}^{c+1}=h_{i j, t l}\left(V_{i,}^{c} \angle \delta_{i}^{c}, V_{j}^{c} \angle \delta_{j}^{c}\right) \\
\rho_{i, t l}^{c+1}=\lambda_{i, t l}^{c}
\end{gathered}
$$

The optimal solution of the initial state estimation problem is obtained if the necessary conditions for optimality 
according to the coordinating variables are fulfilled. This can be checked by calculating of errors

$$
\begin{aligned}
& \varepsilon_{1}=y_{i, i n j}^{c+1}-y_{i, i n j}^{c} \\
& \varepsilon_{2}=\rho_{i, i n j}^{c+1}-\rho_{i, i n j}^{c} \\
& \varepsilon_{3}=y_{i, t l}^{c+1}-y_{i, t l}^{c} \\
& \varepsilon_{4}=\rho_{i, t l}^{c+1}-\rho_{i, t l}^{c}
\end{aligned}
$$

If $\varepsilon_{1} \leq \varphi_{1}, \varepsilon_{2} \leq \varphi_{2}, \varepsilon_{3} \leq \varphi_{3}, \varepsilon_{4} \leq \varphi_{4}$

Where $\varphi_{1}>0, \varphi_{2}>0, \varphi_{3}>0, \varphi_{4}>0$ are very small predefined number, the optimal solution of the coordinating problem and the sub-problems are obtained.

\subsection{Formulation of the State Estimation Sub-Problem of the First Level}

The state estimation problem for every isolated sub-system is formulated using decomposed Lagrangian function for sub-problem criterion given by:

$$
\begin{aligned}
L_{i}= & \frac{\left(V_{i}^{\text {meas }}-V_{i}^{e s t}\right)^{2}}{\sigma_{i, V}^{2}}+ \\
& +\rho_{i, i n j}^{c} y_{i, i n j}^{c}-\sum_{\substack{j=1 \\
j \neq i}}^{N_{S}} \rho_{j i, i n j}^{c} h_{j i, i n j}\left(V_{i} \angle \delta_{i}\right)- \\
& -\lambda_{i, i n j}^{T}\left[-y_{i, i n j}^{c}+z_{i, i n j}-h_{i, i n j}\left(V_{i} \angle \delta_{i}\right)\right]+ \\
& +\rho_{i, t l}^{c} y_{i, t l}^{c}-\rho_{j}^{c} h_{j i}\left(V_{i} \angle \delta_{i}\right)+ \\
& +\lambda_{i, t l}^{T}\left[z_{i, t l}-h_{i, t l}\left(V_{i} \angle \delta_{i}\right)-y_{i, t l}^{c}\right]+ \\
+ & \lambda_{i, V}^{T}\left[z_{i, v}-V_{i}\right]+\lambda_{i, \text { flow }}^{T}\left[z_{i, f l o w}-h_{i, f l o w}\left(V_{i} \angle \delta_{i}\right)\right]
\end{aligned}
$$

\subsubsection{Solution of the First Level Problems}

A Lagrangian function is formed for every sub-problem as follows:

$$
\begin{aligned}
L_{i}^{i} & =\frac{\left(V_{i}^{\text {meas }}-V_{i}^{e s t}\right)^{2}}{\sigma_{i, V}^{2}}+ \\
& +\rho_{i, i n j}^{c} y_{i, i n j}^{c}-\sum_{\substack{j=1 \\
j \neq i}}^{N_{S}} \rho_{j i, i n j}^{c} h_{j i, i n j}\left(V_{i} \angle \delta_{i}\right)- \\
& -\lambda_{i, i n j}^{T}\left[-y_{i, i n j}^{c}+z_{i, i n j}-h_{i, i n j}\left(V_{i} \angle \delta_{i}\right)\right]+ \\
& +\rho_{i, t l}^{c} y_{i, t l}^{c}-\rho_{j}^{c} h_{j i}\left(V_{i} \angle \delta_{i}\right)+ \\
& +\lambda_{i, t l}^{T}\left[z_{i, t l}-h_{i, t l}\left(V_{i} \angle \delta_{i}\right)-y_{i, t l}^{c}\right]+ \\
& +\lambda_{i, V}^{T}\left[z_{i, v}-V_{i}\right]+\lambda_{i, \text { flow }}^{T}\left[z_{i, \text { flow }}-h_{i, \text { flow }}\left(V_{i} \angle \delta_{i}\right)\right]
\end{aligned}
$$

The optimal solution is based on the necessary conditions for optimality as follows:

$$
\begin{aligned}
& \frac{\partial L_{i}^{i}}{\partial V_{i}}=2 \frac{\left(V_{i}^{\text {meas }}-V_{i}\right)}{\sigma_{i, V}^{2}}- \\
& -\sum_{\substack{j=1 \\
j \neq i}}^{N_{S}}\left(\frac{\partial h_{j i, i n j}\left(V_{i} \angle \delta_{i}\right)}{\partial V_{i}}\right)^{T} \rho_{i, i n j}^{c}- \\
& -\left(\frac{\partial h_{i, i n j}\left(V_{i} \angle \delta_{i}\right)}{\partial V_{i}}\right)^{T} \lambda_{i, i n j}- \\
& -\left(\frac{h_{j i, t l}\left(V_{i} \angle \delta_{i}\right)}{\partial V_{i}}\right)^{T} \rho_{j, t l}^{c}- \\
& -\left(\frac{h_{i, t l}\left(V_{i} \angle \delta_{i}\right)}{\partial V_{i}}\right)^{T} \lambda_{i, t l}-\lambda_{i, V}- \\
& -\left(\frac{h_{i, \text { flow }}\left(V_{i} \angle \delta_{i}\right)}{\partial V_{i}}\right)^{T} \lambda_{i, \text { flow }}=0 \\
& \frac{\partial L_{i}^{i}}{\partial \delta_{i}}=-\sum_{\substack{j=1 \\
j \neq i}}^{N_{S}}\left(\frac{\partial h_{j i, i n j}\left(V_{i} \angle \delta_{i}\right)}{\partial \delta_{i}}\right)^{T} \rho_{j, i n j}^{c}- \\
& -\left(\frac{\partial h_{i, i n j}\left(V_{i} \angle \delta_{i}\right)}{\partial \delta_{i}}\right)^{T} \lambda_{i, i n j}- \\
& -\left(\frac{\partial h_{j i, t l}\left(V_{i} \angle \delta_{i}\right)}{\partial \delta_{i}}\right)^{T} \rho_{j, t l}^{c}- \\
& -\left(\frac{h_{i, t l}\left(V_{i} \angle \delta_{i}\right)}{\partial \delta_{i}}\right)^{T} \lambda_{i, t l}- \\
& -\left(\frac{\partial h_{i, \text { flow }}\left(V_{i} \angle \delta_{i}\right)}{\partial \delta_{i}}\right)^{T} \lambda_{i, \text { flow }}=0 \\
& \frac{\partial L_{i}^{i}}{\partial \lambda_{i, i n j}}=z_{i, i n j}-y_{i, i n j}^{c}-h_{i, i n j}\left(V_{i} \angle \delta_{i}\right)=0 \\
& \frac{\partial L_{i}^{i}}{\partial \lambda_{i, t l}}=z_{i, t l}-h_{i, t l}\left(V_{i} \angle \delta_{i}\right)-y_{i, t l}^{c}=0 \\
& \frac{\partial L_{i}^{i}}{\partial \lambda_{i, V}}=z_{i, V}-V_{i}=0 \\
& \frac{\partial L_{i}^{i}}{\partial \lambda_{i, \text { flow }}}=z_{i, \text { flow }}-h_{i, \text { flow }}\left(V_{i} \angle \delta_{i}\right)=0
\end{aligned}
$$

The solution of set of equations (47) to (52) gives the necessary conditions for optimality determines the optimal solution of the ith sub-problem. Eqns (47) t0 (52) are nonlinear with many variables; they cannot be solved by analytical method. Gradient procedures are used to calculate the values of primal variables $\left(V_{i}, \delta_{i}\right)$ and the dual variables $\left(\lambda_{i, i n j}, \lambda_{i, t l}, \lambda_{i, V}, \lambda_{i, f l o w}\right)$ as follows: 


$$
\begin{aligned}
& V_{i}^{t+1}=V_{i}^{t}-\alpha_{i, V} \varepsilon_{i, V} \\
& \delta_{i}^{t+1}=\delta_{i}^{t}-\alpha_{i, \delta} \varepsilon_{i, \delta} \\
& \lambda_{i, i n j}^{t+1}=\lambda_{i, i n j}^{t}+\alpha_{i, i n j} \varepsilon_{i, i n j} \\
& \lambda_{i, t l}^{t+1}=\lambda_{i, t l}^{t}+\alpha_{i, t l} \varepsilon_{i, t l} \\
& \lambda_{i, V}^{t+1}=\lambda_{i, V}^{t}+\alpha_{i, V} \varepsilon_{i, \lambda} \\
& \lambda_{i, \text { flow }}^{t+1}=\lambda_{i, \text { flow }}^{t}+\alpha_{i, \text { flow }} \varepsilon_{i, \text { flow }}
\end{aligned}
$$

where

$$
\varepsilon_{i, V}, \varepsilon_{i, \delta}, \varepsilon_{i, i n j}, \varepsilon_{i, t l}, \varepsilon_{i, \lambda}, \varepsilon_{i, f l o w} \text { are errors and } \alpha_{i} \text { is step- }
$$
length.

The calculations given by eqn (53) are performed under the given by the second level values. The gradient procedure continues until convergence on maximum number of iterations on the first level is attained. Norm [21] of the errors of every iteration is calculated from:

$$
\begin{aligned}
& \varepsilon_{1}^{i}=\left\|\varepsilon_{i, V}\right\| \\
& \varepsilon_{2}^{i}=\left\|\varepsilon_{i, \delta_{i}}\right\| \\
& \varepsilon_{3}^{i}=\left\|\varepsilon_{i, i n j}\right\| \\
& \varepsilon_{4}^{i}=\left\|\varepsilon_{i, t l}\right\| \\
& \varepsilon_{5}^{i}=\left\|\varepsilon_{i, \text { flow }}\right\|
\end{aligned}
$$

Norms of the errors are compared with small pre-defined positive numbers given by the following constants $\phi_{1}, \phi_{2}, \phi_{3}, \phi_{4}, \phi_{5}$. If $\varepsilon_{1}^{i} \leq \phi_{1}, \varepsilon_{2}^{i} \leq \phi_{2}, \varepsilon_{3}^{i} \leq \phi_{3}, \varepsilon_{4}^{i} \phi_{4}, \varepsilon_{5}^{i} \leq \phi_{5}$ is fulfilled, then the optimal solution is attained and the computation process is stopped. When the calculations of the first level are completed, the values of $V_{i} \delta_{i}, \lambda_{i, V} \lambda_{i, t l}$ are sent to the second level and the new values of the coordinating variables are calculated and so on. The computation set up and communication between first level and second level is presented in Figure 3.

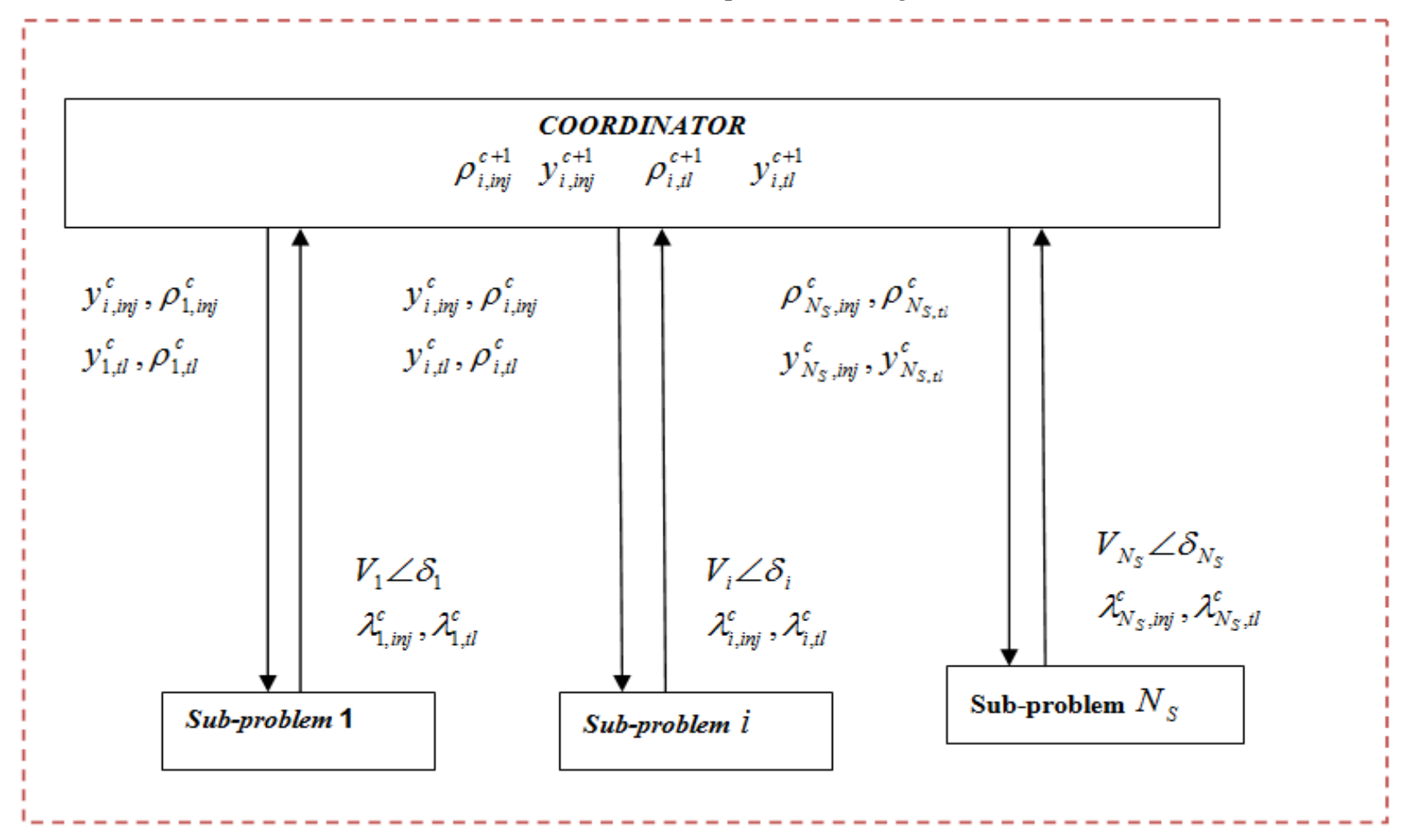

Figure 3. Communication between Two level structures for solution of state estimation problem

\section{Algorithms}

The computation procedure is implemented using the following first and second level algorithms.

\subsection{First Level Algorithm}

At first level, the optimal operating condition of each subsystem is determined by solving independent $\mathrm{N}$ power system state estimation. At this level the interconnection between the sub-systems have not to be considered. Interconnection values are provides by the coordinator.
Hence, gradient methods are used to compute the primal and dual variables of the isolated sub-system. The following algorithm is used to calculate the solution of the first level sub-problem. Before starting the algorithm, first the number of iterations is defined.

I. Initialize $\mathrm{t}=0$

II. Set initial values for $V_{i}, \delta_{i}, \lambda_{i, i n j}, \lambda_{i, \text { flow. }} \lambda_{i, t l}, \lambda_{i, V}$. Initial value of $V_{i}$ is obtained from load flow program and $\delta_{i}$ is set equal to zero.

III. Obtain the values of the coordinating variables 
IV. $\rho_{i, i n j}, \rho_{i, t l}, y_{i, i n j}, y_{i, t l}$ from the second level

V. Improve values of set variables using Eqn (53)

VI. Check for conditions for convergence using Eqn (54)

If conditions of convergence satisfy (54) stop the procedure and sent the values of $V_{i}, \delta_{i}, \lambda_{i, i n j}, \lambda_{i, t l}$ to the second level. If not go to step II. First level algorithm is schematically given in Figure 4.

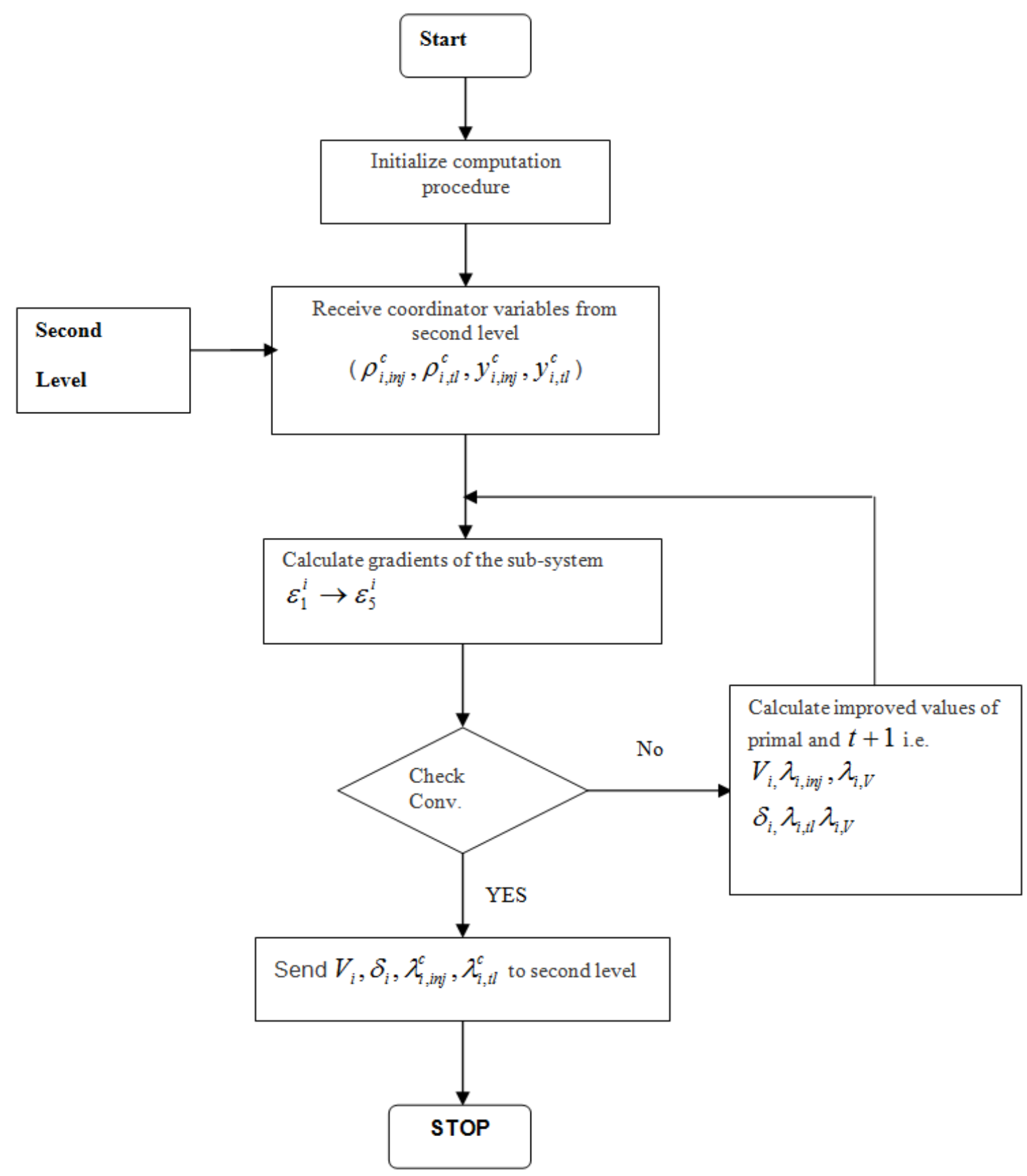

Figure 4. First level algorithm flowchart

\subsection{Second Level Algorithm}

The coordinator does not know or need the detailed operating information of each sub-system. The coordinator executes the following function:

I. Predict the values of Lagrange's variables and interconnection variables and sent predicted variables to the first level

II. Wait all calculations on the first level to be completed and receive the calculated values from first level

III. Compare $\rho_{i, i n j}, \rho_{i, t l}$ if the error between them is bigger than pre-defined tolerance, calculate the improved values of the coordinating variables

IV. If the error is smaller than the pre-defined tolerance, stop the procedure.

It can be observed that the coordinator has little work to do. The coordinator does not calculate the state vector but has to 
predict the Lagrange's variables and variables related to subsystem interconnections. The positive characteristic of this method is that of reducing computation work at coordinator level. The second level algorithm is schematically given in Figure 5.

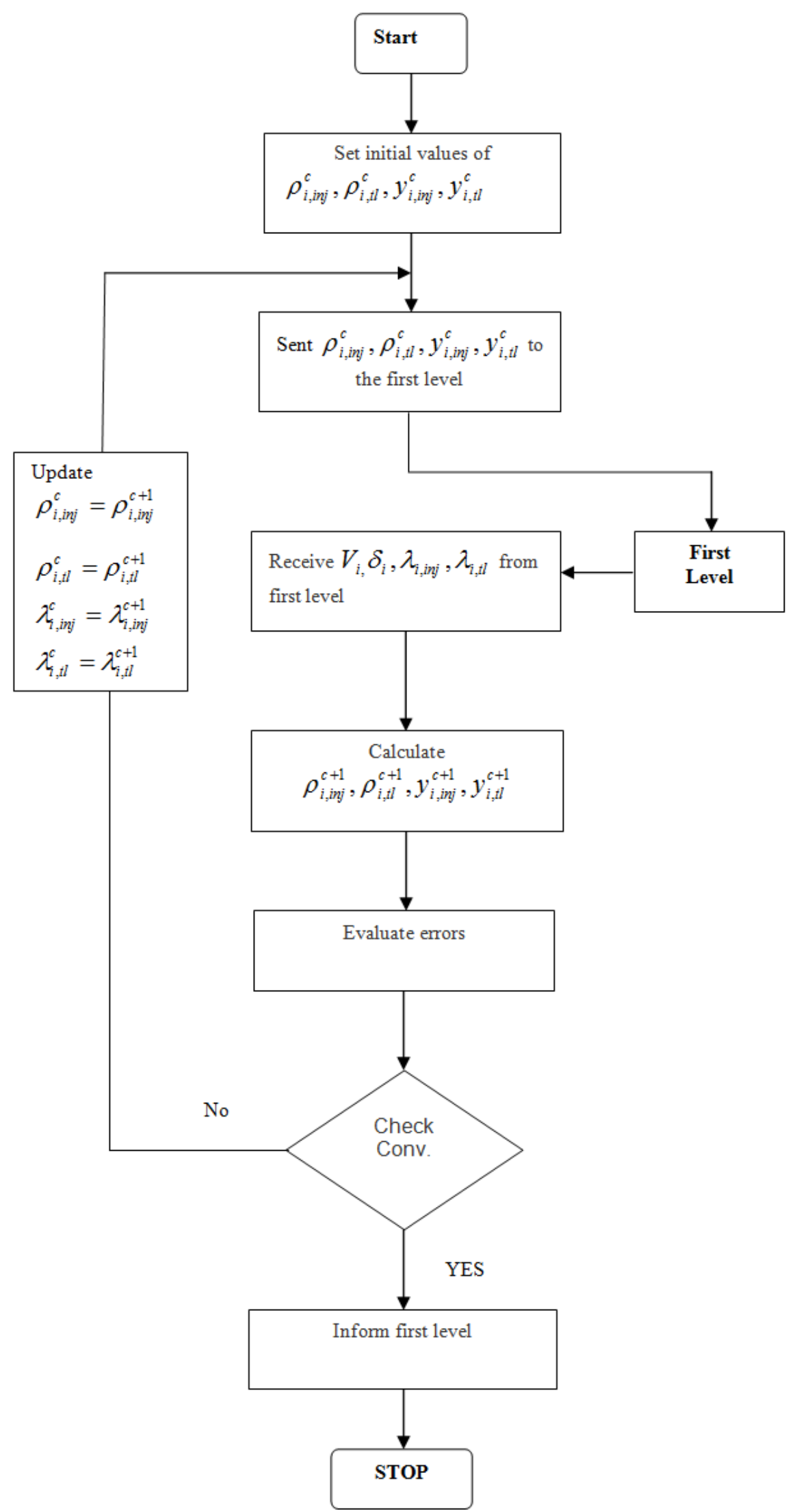

Figure 5. Second level algorithm flowchart 


\section{Discussion}

The developed decomposition-coordination model and algorithm in this paper presents positive characteristic in solving the power system state estimation problem. First, the solution of power system state estimation is reduced to the solution of $\mathrm{N}+1$ independent sub-problem. Secondary, the implementation of the algorithm is carried out using parallel and distributed architectures. In this way, the computational task corresponding to the decomposition work can be carried out by a unique parallel computer located in the power system central dispatch centre, using a cluster or by using a suitable distributed computing scheme with several processors located at lower levels in the control hierarchical such as in the regional control centres.

First and second algorithms are applied in solving the estimation problem provided that: the system is decomposed into sub-systems or areas, at least one generating bus is available in a sub-system and the sub-system is observable i.e. measurements from the sub-system are enough to perform the state estimation.

Decomposition-coordination method and algorithm is aimed at facilitating parallel or distributed processing, decentralizing measurement of a large-scale power system networks, reducing amount of measurements sent to central dispatch centre and reducing computation time and complexity on solution of state estimation.

\section{Conclusion}

In this paper the formulation of the problem for decomposed solution of power system state estimation is presented. Measurement models or voltage magnitude, real and reactive power injections, real and reactive line power flows, and real and reactive power flows in tie-lie lines connecting sub-systems are developed. State estimation problem solution using two-level structure is proposed. First and second level algorithms for implementing the two-level computation are presented. The developed decompositioncoordination method, models and algorithm presents positive characteristic in solving power system state estimation of large-scale power system network and can be implemented using parallel or distributed computing architecture. Further work is still going on to establish the accuracy of the proposed approach. The proposed method will be tested using IEEE 14, IEEE 30, and IEEE 57 buses.

\section{References}

[1] J. Gu, K.A. Clements, G. Krumpholz, P.Davis, “ The solution of ill-conditioned Power System State Estimation Problem via the method of Peters and Wilkinson" Power Industry Computer Applications Conference Proceedings, Huston, May 1983, pp.239-246

[2] A.Monticelli, "State Estimation in Electric Power Systems: A generalized Approach, Kluweri Academic Publishers, Boston,
1999.

[3] A.Bose, and K.A. Clements, "Real-Time modelling of Power Networks", IEEE Proceeding, Special issue on Computers in Power System Operations, Vol., 75, No. 12, Dec 1987, pp. $1607-1622$

[4] B. Stott, O. Alsac and A. Monticelli, "Security Analysis and Optimization", IEEE Proceeding, Vol. 75, No.12, Dec 1987, pp. $1623-1644$

[5] Dy Liacco, "System security: The computer role." IEEE Spectrum, Vol.16, No. 6, June 1978, pp. 48-53

[6] A.A. Hossam-Eldin, E.N. Abdallah and M.S.El-Nozahy, "A modified Genetic Based Technique for solving the Power System State Estimation Problem," World Academy of Science, Engineering and Technology, Vol.55, 2009, pp. 311320 .

[7] J.B. Calvalho and F.M. Barbosa, "A parallel Algorithm to Power System State Estimation ," IEEE, 1988, pp. 1213-1217

[8] O. Alsac, N. Vempati, B. Stott, and A. Monticelli, "Generalized State Estimation," IEEE Transactions on Power Systems, Vol. 13, No.3, August 1998, pp. 1069-1075

[9] F.C. Schweppe, J. Wildes, and D. Rom, "PowerSystemStaticState Estimation Parts: I, II and III," Power Industry Computer Conference, PICA, Denver, Colorado, June 1969.

[10] K.A. Clements and B.F. Wollenberg, "An algorithm for Observability Determination in Power System State Estimation,” Paper A75 447-3, IEEE/PES Summer Meeting, San Francisco, CA, July 1975.

[11] P. Zarco and A.G. Exposito, "Power System Parameter Estimation: A survey," IEEE Trans. on Power Systems, Vol. 15, No.1, Feb. 2000, pp. 216-222

[12] Th. VanCutsem, J.L. Howard, M. Ribben-Pavella and Y.M. ElFattah, "Hierarchical State Estimation," International Journal Of Electric Power and Energy Systems, Vol. 2, April 1980, pp. 70-78

[13] Th. VanCutsem, J.L. Howard, and M. Ribben-Pavella, "A Two-level Static State Estimation for Electric Power Systems," IEEE Trans. on PAS, Vol. PAS, August 1981, pp. 3722-3732

[14] M.Y. Patel and A.A. Girgis, "Two level State Estimation for Multi-area Power System," IEEE Power Engineering Society General Meeting, June 2007

[15] S. Lakshminarasimhan, and A.A. Girgis, "Hierarchical State Estimation Applied to Wide-Area Power Systems," IEEE Power Engineering Society General Meeting, June 2000, pp. $1-6$.

[16] J.A. Aguado, C.P. Molina and V.H. Quintana, "Decentralized PowerSystemState Estimation: A DecompositionCoordination Approach,2 IEEE Porto Power Tech. Conference, $10^{\text {th }}-13^{\text {th }}$ September, 2001, Porto, Portugal, Vol. 3, pp. 6-11

[17] M. Singh and M. Titli, "System: decomposition, Optimization and Control, Kluweri Publishers, Boston, 1978

[18] M.B. Couto Filho, A.M. Leite da Silva and D.M. Falcao, "Bibliography on Power System State Estimation (19681989)", IEEE Trans. on PWR, Vol. 5, No.3, August 1990, pp.950-961 
[19] R.D. Masiello and F.C. Schweppe, "A tracking Static State Estimator,” IEEE, Vol. PAS-90, March/April 1971, pp.10251033

[20] M.A. Kusekwa, "Real-Time State Estimation of a Distributed
Electrical Power System Under Conditions of Deregulation," $\mathrm{PhD}$ Thesis in Electrical Engineering, Cape Peninsula University of Technology, Cape Town, May 2010.

[21] http://www.mathworld.wolfram.com/Norm.html 